Colloque C3, suppl. au Journal de Physique III, Vol. 1, octobre 1991

\title{
THE CONSTITUTIVE MODELING OF POROUS METALS AT HIGH RATES OF DEFORMATION
}

\author{
M. da SILVA and K.T. RAMESH \\ Department of Mechanical Engineering, The Johns Hopkins \\ University, Baltimore, MD 21218, U.S.A
}

\begin{abstract}
The constitutive behavior of fully dense and porous Ti-6Al-4V at high rates of deformation is investigated using a Kolsky compression bar: stress-strain curves are obtained for homogeneous compressive deformations at strain rates between $10^{2}-10^{4} \mathrm{~s}^{-1}$. In addition to the high rate testing, the behavior of the fully dense and porous materials in quasistatic compression is obtained. The porous metal does show a higher degree of strain hardening at both low and high rates due to the compressibility of the pores.
\end{abstract}

\section{Introduction}

Predicting the deformation behavior of sintered powder metals undergoing manufacturing processes requires knowledge of the constitutive law. Such materials exhibit complex thermomechanical behavior that is inherently time dependent and hereditary. This behavior is largely due to the evolution of the porosity with the deformation, which makes inapplicable conventional plasticity theories that are well suited for pore-free metals; in the deformation of porous metals, however, the volume does not remain constant. The titanium alloy chosen for this work is Ti-6Al-4V, a two phase $\alpha-\beta$ alloy which is the most widely used commercial titanium alloy.

Samples of wrought Ti-6Al-4V (fully dense) are used to characterize the mechanical properties of the matrix for a given microstructural state. Then, through a process of heat treatment a microstructure is generated in the fully dense Ti-6 Al-4Vsimilar to that of the matrix of the sintered porous metal. Porosity estimates in terms of pore volume fraction, pore size and mean distribution are made using an image processing system and optical micrograph of representative microsections.

The primary experimental facility used is the Kolsky compression bar (or Split-Hopkinson Pressure Bar). Using standard procedures, flow stress measurements are made using the recorded stress-strain histories. In addition to the high rate testing, the quasi-static behavior (in compression) of the fully dense and porous metals is determined.

\section{Background}

Residual porosity is a major factor influencing the mechanical behavior of sintered metals. The effect of porosity on the mechanical behavior of sintered materials has been investigated theoretically and experimentally by a number of researchers attempting to understand the elastic and plastic behavior of these 
materials. Many factors besides pore-content influence the mechanical behavior; the effects of porosity are best established by holding these other factors constant, as far as possible. This is difficulty to achieve, so that generally a great deal of scatter is found in data in the literature. A comprehensive set of references is available in Haynes ${ }^{[1]}$.

The volumetric deformation of porous materials has been described by various methods ranging from micromechanical modeling to simple phenomenological modeling. Gurson ${ }^{[2]}$ developed approximate yield criteria and flow rules for porous ductile materials, showing the role of the hydrostatic stress, in plastic yield and void growth. Finite element studies of large flow in two dimensional models of porous materials using an elastic plastic description for the matrix material have been presented by Needleman ${ }^{[3]}$. Numerical studies were also done by Mear ${ }^{[4]}$ for an elastic-plastic matrix to examine the macroscopic yield surface for a cubic array of spherical voids. The pore collapse models ${ }^{[5,6]}$ which use spherical pores present an alternative approach to the development of constitutive models for porous metals. Kim ${ }^{[7]}$ studied the response of porous materials under general three dimensional loading conditions where these materials may exhibit strong coupling between volumetric and deviatoric effects.

Progressive densification of sintered powder compacts during deformation has led to studies of the applicability of the theory of plasticity ${ }^{\left[8_{0}, 10\right]}$ to the modeling of these materials. In the case of the onset of plastic deformation in isotropic, wrought and cast materials, the von Mises yield criterion and the PrandtlReuss stress-strain relations apply, but they are inapplicable to compressible (porous) materials. Thus, different yield criteria and stress-strain equations are required for such materials. Moreover, the yield criterion should reduce to the von Mises criterion as the porosity tends to zero. Kuhn et al. ${ }^{[11]}$ and Green ${ }^{[12]}$ independently proposed yield criteria and stress-strain relations for these materials. Both of these theories suggest that the yield criterion is a function of the first invariant of stress $J_{l}$ and the second invariant of deviatoric stress $J_{2}^{\prime}$, that is,

$$
F=\left\{\alpha J_{1}^{2}+\beta J_{2}^{\prime}\right\}^{1 / 2}
$$

and that the stress-strain relations are of the Levy-Mises form i.e.

$$
d \varepsilon_{i}=d \lambda\left(\sigma_{i}-\phi \sigma_{m}\right)
$$

where $\alpha, \beta$ and $\phi$ are functions of the relative density, $d \lambda$ is a non-negative constant, and $\sigma$ the hydrostatic stress and $i$ denotes the principal axes. Shima and $O y a n e^{[8]}$ successfully utilized these relations to explain the deformation behavior of porous (sintered) copper, by experimentally evaluating the functions $\alpha, \beta$ and $\phi$ through simple tension and compression tests. Corapceioglu and $\mathrm{Uz}^{[10]}$ reviewed most of the theories of this type and compared the expressions obtained for $\alpha, \beta$ and $\phi$.

The effect of porosity on yield strength, plastic deformation and work hardening of sintered metal is discussed in Haynes ${ }^{[1]}$. A number of researchers have presented experimental data on and simple analyses of the deformation of porous metals such as copper [8], iron [9] and tungsten (Shipman ${ }^{[13]}$ ) under quasistatic compression and tension. Bourcier et al. ${ }^{[14]}$ reported data for porous Ti-6Al-4V under quasistatic tension and reported the role of voids in fracture on a local scale. In most of these analyses, however, the modeling of the constitutive behavior for the porous metal is similar to that of the fully dense metal. The stress-strain relation for polycrystalline metallic material can generally be represented by a power law equation of the form:

$$
\left(\frac{\sigma}{\sigma_{0}}\right)=\left(\frac{\varepsilon}{\varepsilon_{0}}\right)^{n}
$$


where $n$ is the work hardening exponent, and the entire deformation may be regarded as plastic. Attempts have been made to use relations such as these for the deformation of porous materials, by assuming that $n$ is a function of the relative density. This has worked well for specific cases like porous iron (Kuhn et al.[11]).

A number of questions still remain unanswered. Among these is the modeling of the dynamic behavior of porous metals at high rates $\left(10^{2}-10^{4} \mathrm{~s}^{-1}\right)$. Besides Duffy ${ }^{[15]}$ who attempted to incorporate creep, relaxation and rate sensitive phenomena, very few models or experimental data at high rates are available, and this lack of suitable models for predicting the dynamic behavior of porous metals needs to be addressed.

\section{Material Description}

A micrograph of the sintered Ti-6Al-4V in the as-received condition is shown in Fig.[1]. The slowly cooled, Widmanstatten or basketweave microstructure is apparent, as is the residual porosity. The structure consists of transformed $\beta$, and plate-like $\alpha$. The $\alpha$ phase occupies more than $90 \%$ of the matrix and thus is the most significant phase.

The sintered material was obtained from Industrial Materials Technology, Inc., in two batches. The only difference between the two batches was the sintering temperature. Batch $P 1$ was vacuum sintered at $1177^{\circ} \mathrm{C}$ $\left(2150^{\circ} \mathrm{F}\right.$ ) for four hours and then HIPed (Hot Isostatically Pressed). Batch P2 was manufactured by the same process and for the same time but at a sintering temperature of $1266^{\circ} \mathrm{C}\left(2310^{\circ} \mathrm{F}\right)$. All the results presented here are obtained for samples prepared from Batch P1. Porosity estimates of approximately $8 \%$ are reported from water immersion testing of the samples.

\section{Experimental Procedure}

A technique for the measurement of the properties of materials undergoing homogenous deformations at high rates was developed by Kolsky ${ }^{[16]}$ and is the most widely used testing procedure for dynamic property measurements at strain rates of the order of $10^{2}-10^{4} \mathrm{~s}^{-1}$. The instrument consists of two long metal bars that are designed to remain elastic throughout the test. These bars sandwich a small cylindrical specimen. The end of the incident bar is impacted by a projectile and the compressive wave generated propagates down the bar to the specimen. After several reverberations within the specimen, a transmitted wave is sent into the transmitted bar and a reflected wave back into the incident bar. Strain gages placed on the incident and transmitted bars are used to measure the pulses propagating in the bars. The strain gage signals thus provide the histories of the strain rate and stress within the sample; by combining the two signals it is possible to obtain the stress-strain relation for the specimen material under high rates. Then, assuming that we have homogenous deformation, we can obtain the mean specimen stress and mean specimen surain in terms of the strains in the incident $\left(\varepsilon_{R}\right)$ and transmitted bars $\left(\varepsilon_{T}\right)$ as follows :

$$
\overline{\sigma_{s}}=\frac{E A_{b}}{2 A_{s}} \varepsilon_{T}
$$

where $A_{s}$ is the specimen area; and specimen strain rate as,

$$
\dot{\overline{\varepsilon_{s}}}=\frac{2 c_{0}}{l_{0}} \varepsilon_{R}
$$

from which we obtain the specimen strain as 


$$
\overline{\varepsilon_{s}}=\frac{2 c_{0}}{l_{0}} \int_{0}^{t} \varepsilon_{R} d \tau
$$

where $A_{b}$ and $c_{o}$ represent the bar cross-section area and the bar velocity respectively, and $E$ is the Young's modulus of the bars.

\section{Specimen Preparation}

From the micrograph of the sintered Ti-6Al-4V Fig.[1] one can see that the microstructure consists of $\alpha$ plates oriented along specific directions in the plane of the section. The fully dense material was heat treated to generate a microstructure similar to that which exists in the matrix of the porous metal. This required solution treating the wrought $\mathrm{Ti}-6 \mathrm{Al}-4 \mathrm{~V}$ at $1100^{\circ} \mathrm{C}$ for one hour, furnace cooling to room temperature, and then aging at $705^{\circ} \mathrm{C}$ for two hours and air cooling.

After the heat treatments described the fully dense and the matrix of the porous Ti-6Al-4V possess very similar microstructures. In an attempt to further characterize the microstructure, microhardness measurements are made of each batch. The hardness measured for the fully dense Ti-6Al-4V is $418 \mathrm{VHN}$ (Vickers Hardness Number) and that for the matrix of the porous material is $412 \mathrm{VHN}$. The microstructure is also governed to some extent by the size of the grains present. In the case of the fully dense material the grains were approximately $10-20 \mu \mathrm{m}$ across and about $20-80 \mu \mathrm{m}$ in length. Grain size measurements of the Widmanstatten grain structure present in the porous material yielded grains with aspect ratios varying from 2 to as high as 10 in some cases. These grains are actually plate-like structures with lengths between 20 $100 \mu \mathrm{m}$. The measurement of the grain sizes of the two batches is seen to be almost the same $(10-15 \mu \mathrm{macross}$ and $20-80 \mu \mathrm{m}$ in length). Another characteristic feature of these plates is that they seem to be oriented along specific directions.

The other dominant features of the microstructure are the pore volume fraction and the size and aspect ratio of the pores. An image processing system was developed for quantitative analysis of the microstructure of the porous Ti-6Al-4V. By digitizing micrographs of carefully polished microsections and utilizing the fundamental relations of stereology these microstructural parameters can be obtained. It is sufficient to measure the areal pore fraction in order to obtain the pore volume fraction present in the solid. The resulting mean pore area fraction or relative porosity is found to be $7.6 \%$ which is close to the value of $8 \%$ obtained from fluid immersion tests. In measuring the size and shape of the pores present, the image analysis software attempts to fit an ellipse to the pores and then sizes the pore using the major and minor axes of the fitted

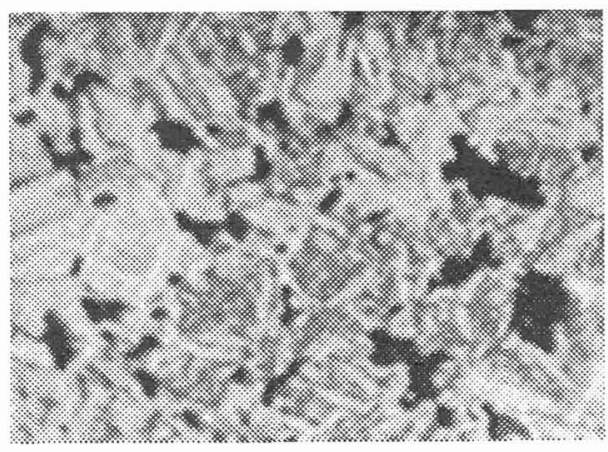

Fig.1. Microstructure of Porous Ti-6Al-4V

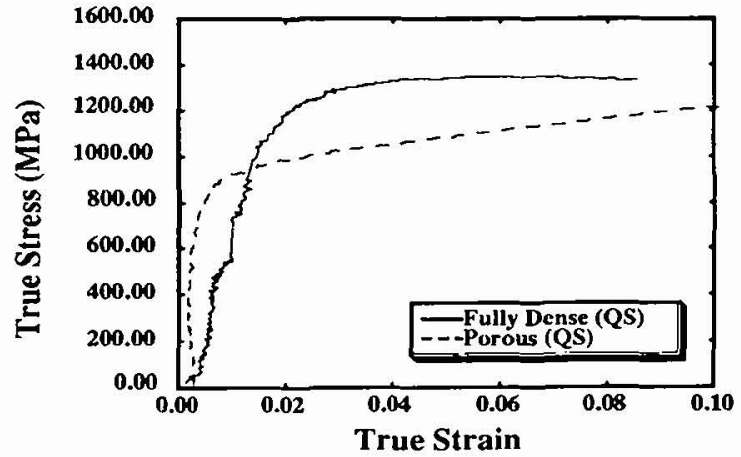

Fig.2. True Stress-Strain in Quasistatic Compression 
ellipse. Using this method the measured values of the major and minor axes yielded a mean aspect ratio of 2.0 for the pores with the mean major axis length equal to $9.46 \mu \mathrm{m}$ and the mean minor axis equal to $4.71 \mu \mathrm{m}$.

\section{Results and Discussion}

The porous and wrought Ti-6Al-4V were subjected to quasistatic compression at strain rates between $10^{-4}-10^{-3} \mathrm{~s}^{-1}$ and the resulting true stress-strain curves are shown in Fig.[2]. These tests are validated by comparing the observed values of the yield strength and modulus with the expected values (Donachie ${ }^{[17]}$ ). The observed $0.2 \%$ yield strength in compression is $845 \mathrm{MPa}$ which compares favorably to the vendor supplied value of $815 \mathrm{MPa}$. A comparison of the quasistatic behavior of the two batches is quite interesting from a number of stand points. The fully dense metal is seen to exhibit a small degree of hardening as compared to the porous metal. The relative increase of the stress level in the case of the fully dense metal over that in the porous metal for identical strains is seen to be of the order of $200 \mathrm{MPa}$. This effect is probably due to the compressibility of the pores and this reasoning is further pursued in analyzing the dynamic tests as well. An important point to note is that the values on this curve correspond to the true stress and strain. This is possible only if another strain measure (such as radial strain) is recorded or the evolution of the porosity with deformation is known, because the porous material cannot be assumed to be incompressible.

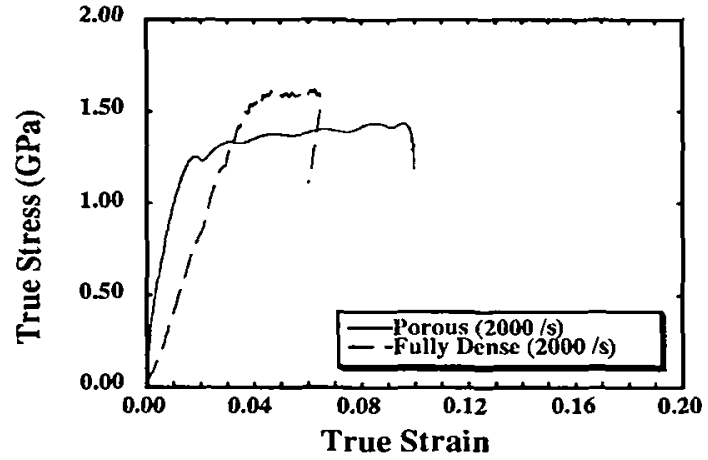

Fig.3. True Stress-Strain at $2000 \mathrm{~s}^{-1}$

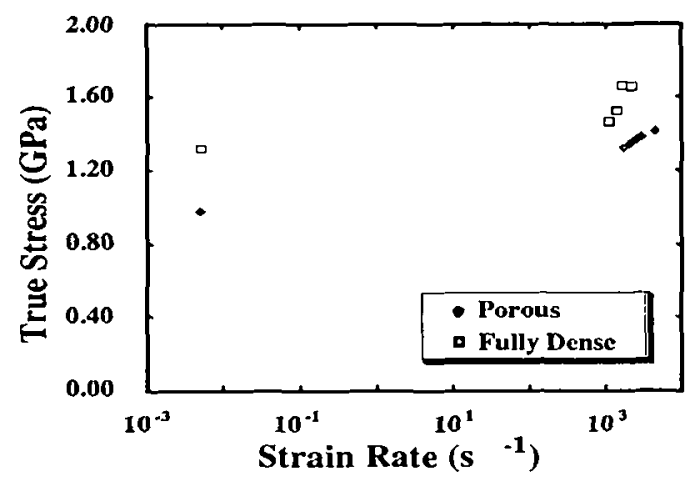

Fig.4. Rate Sensitivity of Porous and Wrought Ti-6Al-4V

The porous material was tested at different rates in the range from $10^{3}-5 \times 10^{3} \mathrm{~s}^{-1}$ using the Kolsky bar. In Fig.[3] the stress-strain behavior of the porous and wrought $\mathrm{Ti}-6 \mathrm{Al}-4 \mathrm{~V}$ are shown at shown at a strain rate close to $2000 \mathrm{~s}^{-1}$. The porous material is seen to harden much more than the wrought alloy at similar strain rates although the wrought metal can sustain a much higher stress level. Also Fig. [4] is a plot of the rate sensitivity of the stress as a function of the strain rate, at a strain of $4 \%$. The fully dense Ti- $6 \mathrm{Al}-4 \mathrm{~V}$ shows appreciable rate sensitivity at high rates which has been reported before for this alloy (Meyer ${ }^{[18]}$, Wulf ${ }^{[19]}$ ). The porous metal is also seen to be quite rate sensitive.

The stress-strain curves used thus far for the wrought metal have utilized values of true stress and strain that are defined by (in usual notation):

$$
\begin{gathered}
\sigma_{\imath}=\sigma(1-\varepsilon) \\
\varepsilon_{\imath}=\ln \left(\frac{l}{l_{0}}\right)=\ln (1-\varepsilon)
\end{gathered}
$$

where $\sigma$ and $\varepsilon$ are the engineering stress-strain values and the subscript $t$ denotes the true values of these 
quantities.

In the case of the porous material this does not represent the true stress and strain state in the material. Since we have material porosity as an additional variable we need another strain measure in order to obtain the evolution of the density (or porosity) as a function of the compressive strain.

The procedure to obtain this evolution of the material porosity involved straining of the specimen at a particular rate (in this case all the tests were performed at a nominal strain rate of $2000 \mathrm{~s}^{-1}$ ) to various amounts of plastic strain (Fig.[5]). The relative density was then measured via two methods, one which involved measuring both mass and volume of the specimens and comparing the density of the porous material with that of the fully dense material. The other method employs Archimedes principle and the procedure outlined by Bourcier et al. ${ }^{[14]}$. The evolution of the relative density of the porous material as a function of the compressive strain is shown in Fig.[6]. The behavior of the density evolution curve at this rate shows almost the same trend as those observed in porous copper (Shima and Oyane ${ }^{[8]}$ ) and for porous iron by (Kim et al. $\left.{ }^{[9]}\right)$. However one must remember that the above authors reported these results from quasistatic tests rather than dynamic tests.

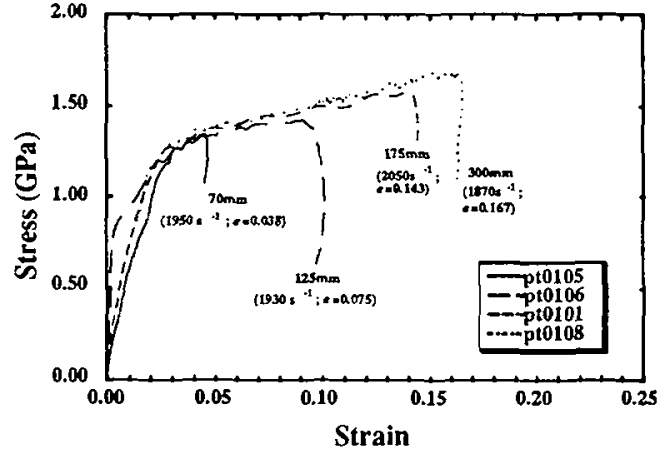

Fig. 5. Stress-Strain curves to obtain Porosity Evolution

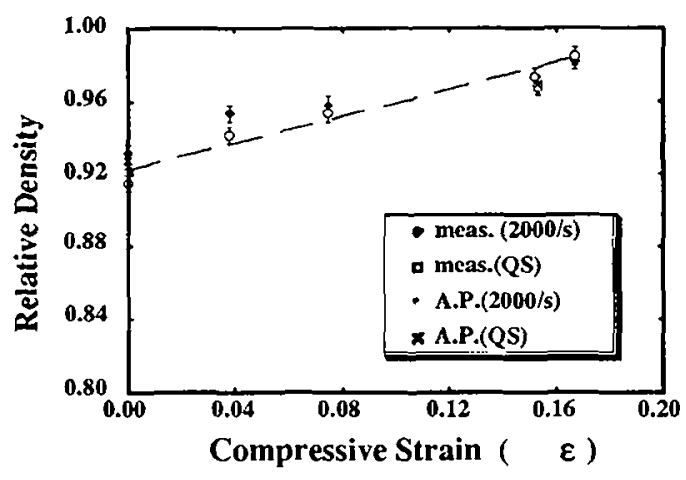

Fig. 6. Density Evolution With Compressive Strain

In order to look at the effect of the strain rate on the evolution of the density (or porosity) similar measurements were made on samples of the porous metal deformed under quasistatic rates and the final density is plotted in Fig.[6]. The results show that the effect of strain rate is almost negligible considering that the quasistatic data points almost coincide with the curve fitted through the high strain rate data.

To obtain the relation between the relative density and the compressive strain (Fig.[6]) a curve is fitted through the data points obtained such that the error involved in the fit is less than $2 \%$. The curve is exponential in nature and approaches the matrix density for large compressive strains. The evolution of the relative density of the sample with compressive strain $\varepsilon$ is described by :

$$
\frac{\rho}{\rho_{m}}=\left(\frac{\rho_{0}}{\rho_{m}}\right) \exp (a \varepsilon)
$$

where $\rho_{0}$ is the initial density of the porous material, $\rho_{m}$ is the density of the matrix and $a$ is a fitted constant. The values of $a$ is ; $a=0.3904$ for the case where the density is measured using Archimedes Principle. The true stress and true strain values can now be computed for the porous material as follows : 


$$
\begin{aligned}
& \sigma_{t}=\left(\frac{P}{A_{0}}\right)(1-\varepsilon)\left(\frac{\rho}{\rho_{0}}\right) \\
& \varepsilon_{\mathrm{t}}=\ln \left[(1-\varepsilon)\left(\rho / \rho_{0}\right)\right]
\end{aligned}
$$

It is this true stress-strain curve for the porous material that is compared with that of a similar curve for the fully dense batch for quasistatic compression in Fig.[2] and at a rate of $2000 \mathrm{~s}^{-1}$ in Fig.[3]. These figures show that the porous material does harden more than the fully dense material at both strain rates.

Another important feature noticeable in Fig.[2] is that the stress-strain curve for fully dense metal reaches a peak value at a strain of $8 \%$. This corresponded to failure of the specimen which was observed to shear across at an angle to the loading axis. This appears to be consistent for such a microstructure (Donachie ${ }^{[17]}$ ) which has a much higher yield strength $(950 \mathrm{MPa})$ and a very low percentage elongation however when this behavior is compared to that of the porous metal the differences are quite large. For instance the porous material was deformed up to a strain of $15 \%$ at similar rates with no indication of macroscopic failure. However it is entirely possible that due the high stresses associated with the shape of the pores there is going to be localized shear banding interconnecting individual pores. This was observed by Vedula and Heckel ${ }^{[20]}$ in the case of porous iron (for $6 \%$ porosity).

When these two materials undergo homogenous deformations at high rates the general behavior of both materials is essentially the same as that manifested in the case of their respective quasistatic stress-strain behavior. This is shown in Fig. [3] which is a typical stress-strain curve at a rate of $2000 \mathrm{~s}^{-1}$ for both materials. The primary difference however is depicted in Fig.[4] which shows the strong rate sensitivity of the flow stress of the wrought and porous Ti-6Al-4V to the natural strain rate. The effect of annealing above the $\beta$ transus temperature results in increased strength accompanied with a loss of ductility which is observed in the dynamic response of the fully dense metal. The rise in stress level corresponds to the levels observed in Fig.[4]. The other result of this particular heat treatment of the fully dense metal is the low ductility of the alloy. In the dynamic tests at strain rates between $800-2500 \mathrm{~s}^{-1}$ this loss of ductility was clearly observable as it was impossible to generate higher strains than $7 \%$ without observing bands (what appears to be the edge of a shear band) or causing fracture within the sample.

The high rate behavior of the porous material is similar to that observed at very low rates in terms of the general behavior of the stress-strain curve. The amount of strain achievable in the porous metal before failure is approximately three or four times larger than that observed in the fully dense material even at high rates. The highest strain observed in any of the tests on the porous material is $20.2 \%$ and even here there seems to be no indication of failure in the specimen in general.

\section{Conclusions}

The Kolsky Compression bar is an effective instrument with which to study the influence of material porosity on the dynamic response of Ti-6Al-4V. The behavior of the porous metal is significantly different than that of fully dense Ti-6Al-4V which is heat treated so that the microstructure of the fully dense metal is similar to that of the matrix of the porous $\mathrm{Ti}-6 \mathrm{Al}-4 \mathrm{~V}$. Among the more noticeable effects are a reduction in elastic modulus and strength. Further, the porous metal is observed to have a much higher degree of hardening. Another effect of material porosity observed in the tests is the stabilizing influence of the pores in uniaxial compression suppressing the localized deformation observed in the fully dense material. 
Porosity is seen to increase the ductility of Ti-6Al-4V (for the particular microstructure studied) considerably.

\section{References}

1. Haynes, R. (1981) The Mechanical Behavior of Sintered Metals, Freund, London.

2. Gurson, A.L., (1977), Jour. of Engg. Mater. and Tech., pp. 2-15.

3. Needleman, A., (1972), J. of App. Mech., Vol. 39, pp. 964-970.

4. Mear, M.E., (1990), Mech. of Mater., Vol. 9, pp. 33-48.

5. Caroll, M.M. \& Holt, A.C., (1973), J. App. Phys., Vol. 44, pp. 4388.

6. Kim, K.T. \& Caroll, M.M., (1987), Int. J. Plasticity, Vol. 3, pp. 63.

7. Kim, K.T., (1988), Int. J. of Solids and Struct., Vol. 24, No. 9, pp. 937-945.

8. Shima, S. \& Oyane, M., (1976), Int. Jour. Mech. Sci., Vol. 18, pp. 285-291.

9. Kim, K.T., Suh, J. \& Kwon, Y.S., (1990), Private Communication.

10. Corapcioglu, Y. \& Uz, T., (1978), Powder Tech., Vol. 21, pp. 269-274.

11. Kuhn, H.A. \& Downey, C.L., (1971), Int. J. Powder Metall., Vol. 7, (1), pp. 15.

12. Green, R.J., (1972), Int. Jour. Mech. Sci., Vol. 14, , pp.215-224.

13. Shipman, F.H. et al., (1975), Report No. 75-79, Salt Lake City, Utah.

14. Bourcier, R.J., et al., (1986), Acta Metall., 34, No. 12, pp. 2443-2453.

15. Duffy, S.F., (1988), Mech. of Mater., Vol. 7, pp. 245-254.

16. Kolsky, H., (1949), Proc. Phys. Soc. London, Vol.62B, pp. 676-700.

17. Donachie Jr. (ed.), M.J., (1982), Titanium and Titanium Alloys - Source Book, ASM.

18. Meyer, L.W., (1984) Deutsche Gessellschaft fur Metallkunde, Ed. Lutjering, G., Zwicker, U. and Bunk, W., pp. 1851-1858.

19. Wulf, G. L., (1979), Int. J. of Mech. Sci., Vol. 21, pp. 713-718.

20. Vedula, K.M. \& Heckel, R.W., (1981), in Modern Developments in Powder Metallurgy. p 759. 